\title{
Colored Prüfer codes for $k$-edge colored trees
}

\author{
Manwon Cho, Dongsu Kim*广 Seunghyun Seo and Heesung Shin \\ Department of Mathematics, KAIST, Daejeon 305-701, Korea
}

Submitted: Dec 31, 2002; Accepted: Oct 15, 2003; Published: Jul 19, 2004

MR Subject Classifications: 05C05, 05C30

\begin{abstract}
A combinatorial bijection between $k$-edge colored trees and colored Prüfer codes for labelled trees is established. This bijection gives a simple combinatorial proof for the number $k(n-2) !\left(\begin{array}{c}n k-n \\ n-2\end{array}\right)$ of $k$-edge colored trees with $n$ vertices.
\end{abstract}

\section{Introduction}

A $k$-edge colored tree is a labelled tree whose edges are colored from a set of $k$ colors such that any two edges with a common vertex have different colors [2, p81, 5.28]. For a pair $(n, k)$ of positive integers, let $\mathcal{C}_{n, k}$ denote the set of all $k$-edge colored trees on vertex set $[n]=\{1,2, \ldots, n\}$, with color set $[k]$. The number of $k$-edge colored trees in $\mathcal{C}_{n, k}$ is already known:

Theorem 1. The number of $k$-edge colored trees on vertex set $[n], n \geq 2$, is

$$
k(n k-n)(n k-n-1) \cdots(n k-2 n+3)=k(n-2) !\left(\begin{array}{c}
n k-n \\
n-2
\end{array}\right) .
$$

Stanley in $[2, \mathrm{p} 124]$ introduces a proof of the above formula and asks whether there is a simple bijective proof. In this paper we provide a combinatorial bijection between $k$-edge colored trees and 'colored Prüfer codes', thus establishing a simple bijective proof of the above formula.

The Prüfer code $\varphi(T)=\left(a_{1}, \ldots, a_{n-2}, 1\right)$ of a labelled tree $T$ with vertex set $[n]$ is obtained from the tree by successively pruning the leaf with the largest label. To obtain the code from $T$, we remove the largest leaf in each step, recording its neighbor $a_{i}$, from the tree, until the single vertex 1 is left. The inverse of $\varphi$ can be described easily. Let $\sigma=\left(a_{1}, \ldots, a_{n-2}, 1\right)$ be a sequence of positive integers with $a_{i} \in[n]$ for all $i$. We can find the tree $T$ whose code is $\sigma$ as follows:

${ }^{*}$ Corresponding author: dskim@math.kaist.ac.kr

${ }^{\dagger}$ Partially supported by the Korea Research Foundation Grant(KRF-2001-015-DP0055). 
- Let $V=\{1\}$ and $E=\emptyset$.

- For each $i$ from $n-2$ to 1 ,

- if $a_{i} \notin V$, then set $b_{i+1}=a_{i}$,

- otherwise set $b_{i+1}=\min \{x: x \in[n] \backslash V\}$;

- set $V:=V \cup\left\{b_{i+1}\right\}$ and $E:=E \cup\left\{\left\{a_{i+1}, b_{i+1}\right\}\right\}$.

- Let $b_{1}$ be the unique element in $[n] \backslash V$.

- Finally, set $V:=V \cup\left\{b_{1}\right\}$ and $E:=E \cup\left\{\left\{a_{1}, b_{1}\right\}\right\}$.

- Let $T$ be the tree with vertex set $V$ and edge set $E$.

Example. Let $T$ be the tree in Figure 1. The Prüfer code of $T$ is $(1,6,1,3,3,1)$. We

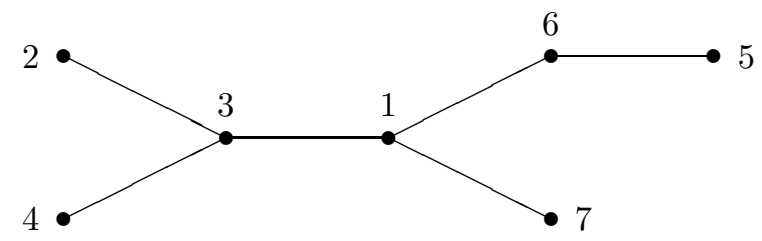

Figure 1: The tree $T$ corresponding to $(1,6,1,3,3,1)$

can recover $T$ from its Prüfer code by the above algorithm.

Clearly, Prüfer codes are in one-to-one correspondence with labelled trees. The following is a well known result. See [1,2].

Theorem 2. The number of the tree on $[n]$ vertices is $n^{n-2}$.

Proof. Any sequence $\left(a_{1}, a_{2}, \ldots, a_{n-2}\right) \in[n]^{n-2}$ of integers corresponds to a Prüfer code $\left(a_{1}, a_{2}, \ldots, a_{n-2}, 1\right)$ which in turn determines a unique labelled tree with vertex set $[n]$.

\section{Colored Prüfer code}

Let $\mathcal{P}_{n, k}$ denote the set of all arrays of the form

$$
\left(\begin{array}{ccccc}
a_{1} & a_{2} & \cdots & a_{n-2} & 1 \\
c_{1} & c_{2} & \cdots & c_{n-2} & c_{n-1}
\end{array}\right)
$$

such that $\left(a_{1}, c_{1}\right),\left(a_{2}, c_{2}\right), \ldots,\left(a_{n-2}, c_{n-2}\right) \in[n] \times[k-1]$ are distinct and $c_{n-1} \in[k]$. An array like the above is called a colored Prüfer code, since its first row is a Prüfer code and its second row can be interpreted as an edge-coloring. 
Lemma 3. The cardinality of $\mathcal{P}_{n, k}$ is

$$
k(n-2) !\left(\begin{array}{c}
n k-n \\
n-2
\end{array}\right) .
$$

Proof. Consider an element $\sigma \in \mathcal{P}_{n, k}$ :

$$
\sigma=\left(\begin{array}{ccccc}
a_{1} & a_{2} & \cdots & a_{n-2} & 1 \\
c_{1} & c_{2} & \cdots & c_{n-2} & c_{n-1}
\end{array}\right) .
$$

The conditions for $\sigma$ are: $\left(a_{i}, c_{i}\right) \in[n] \times[k-1]$ for $1 \leq i \leq n-2, c_{n-1} \in[k]$ and the first $n-2$ columns of $\sigma$ are distinct. So the number of possible $\sigma$ is

$$
k(n k-n)(n k-n-1)(n k-n-2) \cdots(n k-2 n+3)=k(n-2) !\left(\begin{array}{c}
n k-n \\
n-2
\end{array}\right) .
$$

Recall that $\mathcal{C}_{n, k}$ is the set of all $k$-edge colored trees on vertex set $[n]$ with color set $[k]$. Let $T$ be a $k$-edge colored tree in $\mathcal{C}_{n, k}$ with vertex set $V(T)$ and edge set $E(T)$. Let $C_{T}: E(T) \rightarrow[k]$ denote the edge-coloring of $T$, i.e. $C_{T}(e)$ is the color of edge $e$ in $T$.

For each pair of distinct edges $e$ and $e^{\prime}$ in $T$, define the distance between $e$ and $e^{\prime}$, denoted by $d\left(e, e^{\prime}\right)$, to be $l-1$ when $l$ is the shortest length of paths containing $e$ and $e^{\prime}$. Note that the distance between edges sharing a vertex is one.

When $x$ is the smallest neighbor of 1 in $T$, we call the edge $\alpha=\{1, x\}$ the root edge of $T$. For any two edges $e, e^{\prime}$ in $T$ with a common vertex, we call $e$ the parent edge of $e^{\prime}$ and $e^{\prime}$ the child edge of $e$, if $d(e, \alpha)+1=d\left(e^{\prime}, \alpha\right)$.

Let $\widetilde{\mathcal{C}}_{n, k}$ denote the set of labelled trees with vertex set $[n]$ whose edges are colored from a set of $k$ colors, say $[k]$, in such a way that

1. the root edge is colored from $[k]$,

2. any pair of edges sharing a vertex with a common parent edge have distinct colors, and

3. edges which are not the root edge are colored from $[k-1]$.

For a tree $T$ in $\widetilde{\mathcal{C}}_{n, k}$, let $\widetilde{C}_{T}$ denote the edge-coloring of $T$, i.e. $\widetilde{C}_{T}(e)$ is the color of edge $e$ in $T$.

\section{Bijection $\phi$}

We define a mapping $\phi: \widetilde{\mathcal{C}}_{n, k} \rightarrow \mathcal{P}_{n, k}$ through the following steps:

- Set $T_{0}:=T$. 
- For any $i, 1 \leq i \leq n-1$, assuming that $T_{i-1}$ is defined already, define $a_{i}, b_{i}, c_{i}$ and $T_{i}: b_{i}$ is the largest leaf in $T_{i-1}, a_{i}$ is the vertex adjacent to $b_{i}, T_{i}$ is the tree obtained by removing the vertex $b_{i}$ and the edge $\left\{a_{i}, b_{i}\right\}$ from $T_{i-1}$, and $c_{i}=\widetilde{C}_{T}\left(\left\{a_{i}, b_{i}\right\}\right)$.

- Define $\phi(T)$ by

$$
\phi(T)=\left(\begin{array}{ccccc}
a_{1} & a_{2} & \cdots & a_{n-2} & 1 \\
c_{1} & c_{2} & \cdots & c_{n-2} & c_{n-1}
\end{array}\right)
$$

Note that the first row of $\phi(T)$ is the Prüfer code of $T$, so $\phi$ is one-to-one.

Clearly, the first $n-2$ columns of $\phi(T)$ are distinct, and $c_{i} \in[k-1]$ for $1 \leq i \leq n-2$, $c_{n-1} \in[k]$. So $\phi(T)$ is an element in $\mathcal{P}_{n, k}$.

\section{Bijection $\psi$}

We now define a mapping $\psi: \mathcal{P}_{n, k} \rightarrow \widetilde{\mathcal{C}}_{n, k}$, which is the inverse of $\phi$. Let $\sigma$ be an element in $\mathcal{P}_{n, k}$ :

$$
\sigma=\left(\begin{array}{ccccc}
a_{1} & a_{2} & \cdots & a_{n-2} & 1 \\
c_{1} & c_{2} & \cdots & c_{n-2} & c_{n-1}
\end{array}\right)
$$

We construct, by the following algorithm, a labelled tree whose Prüfer code is the first row of $\sigma$, with an edge-coloring $\widetilde{C}_{T}$ :

- Let $V=\{1\}$ and $E=\emptyset$.

- For each $i$ from $n-2$ to 1 ,

- if $a_{i} \notin V$, then set $b_{i+1}=a_{i}$

- otherwise set $b_{i+1}=\min \{x: x \in[n] \backslash V\}$;

- set $V:=V \cup\left\{b_{i+1}\right\}$ and $E:=E \cup\left\{\left\{a_{i+1}, b_{i+1}\right\}\right\}$.

- Let $b_{1}$ be the unique element in $[n] \backslash V$.

- Finally, set $V:=V \cup\left\{b_{1}\right\}$ and $E:=E \cup\left\{\left\{a_{1}, b_{1}\right\}\right\}$.

- Let $T$ be the tree with vertex set $V$ and edge set $E$.

- Set $\widetilde{C}_{T}\left(\left\{a_{i}, b_{i}\right\}\right)=c_{i}$ for $i \in[n-2]$ and $\widetilde{C}_{T}\left(\left\{1, b_{n-1}\right\}\right)=c_{n-1}$.

Let $\psi(\sigma)$ be the resulting tree with edge-coloring $\widetilde{C}_{T}$. Clearly $\psi(\sigma)$ is in $\widetilde{\mathcal{C}}_{n, k}$ and $\psi$ is the inverse of $\phi$. So we have the following.

Lemma 4. The mapping $\phi: \widetilde{\mathcal{C}}_{n, k} \rightarrow \mathcal{P}_{n, k}$ is a bijection and thus the cardinality of $\widetilde{\mathcal{C}}_{n, k}$ is

$$
k(n-2) !\left(\begin{array}{c}
n k-n \\
n-2
\end{array}\right) .
$$




\section{Main result}

We now define a mapping $\Delta$ from $\mathcal{C}_{n, k}$ to $\widetilde{\mathcal{C}}_{n, k}$. For any $T \in \mathcal{C}_{n, k}$, define $\widetilde{C}_{T}: E(T) \rightarrow[k]$ as follows:

- Let $x$ be the smallest neighbor of 1 and $\alpha$ denote edge $\{1, x\}$. Set $\widetilde{C}_{T}(\alpha)=C_{T}(\alpha)$.

- Assume that $\widetilde{C}_{T}(f)$ is defined for all edges $f$ such that $d(\alpha, f)<i$. For an edge $g$ with $d(\alpha, g)=i$, let $h$ be the unique edge such that $d(\alpha, h)=i-1$ and $d(h, g)=1$. Define $\widetilde{C}_{T}(g)$ by

$$
\widetilde{C}_{T}(g)= \begin{cases}C_{T}(g), & \text { if } C_{T}(g) \leq \widetilde{C}_{T}(h) \\ C_{T}(g)-1, & \text { otherwise }\end{cases}
$$

Note that $\widetilde{C}_{T}(f) \leq k-1$ for all $f \neq \alpha$. Let $\Delta(T)$ be the tree $T$ with its edge-coloring $C_{T}$ replaced by $\widetilde{C}_{T}$. Clearly $\Delta(T)$ is an element in $\widetilde{\mathcal{C}}_{n, k}$.

We next define a mapping $\Lambda$ from $\widetilde{\mathcal{C}}_{n, k}$ to $\mathcal{C}_{n, k}$. For any $T \in \widetilde{\mathcal{C}}_{n, k}$, define $C_{T}: E(T) \rightarrow[k]$ as follows:

- Let $x$ be the smallest neighbor of 1 and $\alpha$ denote the edge $\{1, x\}$. Set $C_{T}(\alpha)=$ $\widetilde{C}_{T}(\alpha)$.

- Assume that $C_{T}(f)$ is defined for all edges $f$ such that $d(\alpha, f)<i$. For an edge $g$ with $d(\alpha, g)=i$, let $h$ be the unique edge such that $d(\alpha, h)=i-1$ and $d(h, g)=1$. Define $C_{T}(g)$ by

$$
C_{T}(g)= \begin{cases}\widetilde{C}_{T}(g), & \text { if } \widetilde{C}_{T}(g)<C_{T}(h) \\ \widetilde{C}_{T}(g)+1, & \text { otherwise }\end{cases}
$$

Note that $C_{T}(f) \leq k$ for all $f$ and no pair of two edges with a common vertex have the same color. Let $\Lambda(T)$ be the tree $T$ with its edge-coloring $\widetilde{C}_{T}$ replaced by $C_{T}$. Clearly $\Lambda(T)$ is an element in $\mathcal{C}_{n, k}$.

Clearly, $\Lambda$ is the inverse of $\Delta$. Hence we have the following crucial lemma:

Lemma 5. The mapping $\Delta: \mathcal{C}_{n, k} \rightarrow \widetilde{\mathcal{C}}_{n, k}$ is a bijection.

Example. A $k$-edge colored tree $T$ in $\mathcal{C}_{10,5}$ and its $\Delta(T)$ are in Figures 2 and 3 . The edge $\{1,3\}$ is the root edge.

We can now count the number of the $k$-edge colored trees with $n$ vertices. The following is the restatement of Theorem 1.

Theorem 6 (Main theorem). The number of $k$-edge colored trees on $[n]$ is

$$
k(n-2) !\left(\begin{array}{c}
n k-n \\
n-2
\end{array}\right) .
$$




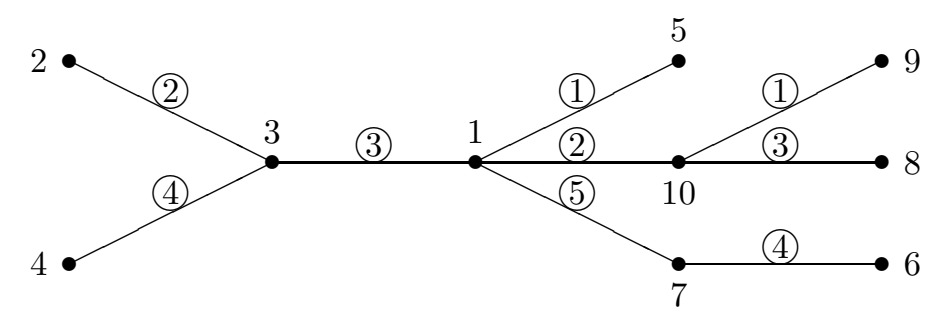

Figure 2: A $k$-edge colored tree $T$ in $\mathcal{C}_{10,5}$

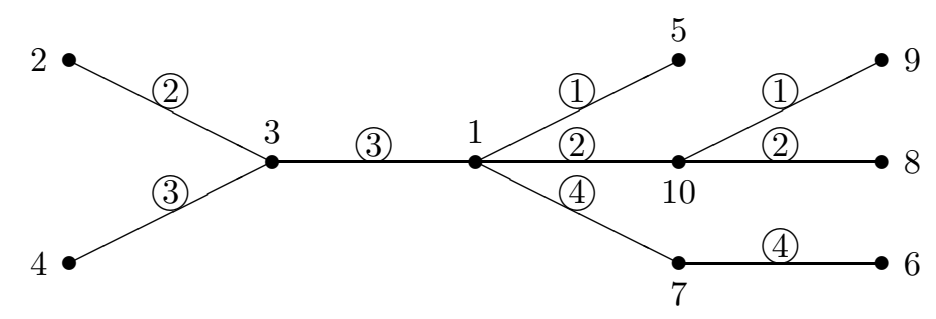

Figure 3: $\Delta(T)$ in $\widetilde{\mathcal{C}}_{10,5}$, i.e. $T$ with $\widetilde{C}_{T}$

Proof. Since $\Delta: \mathcal{C}_{n, k} \rightarrow \widetilde{\mathcal{C}}_{n, k}$ and $\phi: \widetilde{\mathcal{C}}_{n, k} \rightarrow \mathcal{P}_{n, k}$ are bijections, it follows from Lemma 3 or 4 .

The colored Prüfer codes can be used to count certain sets of labelled trees with edgecoloring. Recall that a $k$-edge colored tree is a labelled tree whose edges are colored from a set of $k$ colors such that any two edges with a common vertex have different colors. We now consider slightly different edge-colorings of labelled trees.

Theorem 7 . The number of different labelled trees with vertex set $[n]$ whose edges are colored from a set of $k$ colors in such a way that the color of each edge is different from that of its parent edge is

$$
k(n k-n)^{n-2} .
$$

Proof. Let $T$ be a tree with the property in the statement. Following the steps for the definition of $\phi$, we can obtain an array $\sigma$ corresponding to $T$ :

$$
\sigma=\left(\begin{array}{ccccc}
a_{1} & a_{2} & \cdots & a_{n-2} & 1 \\
c_{1} & c_{2} & \cdots & c_{n-2} & c_{n-1}
\end{array}\right) .
$$

There are $k$ possible ways to choose the $c_{n-1}$. Next, the number of possible ways to choose the $(n-2)$-th column of $\sigma$ is $n(k-1)$, since the color of an edge is different from that of its parent edge. The $i$-th column of $\sigma$ has always $n(k-1)$ choices. Hence the number of such trees is $k(n k-n)^{n-2}$.

Note that the above theorem can be proved by using a generalization of $\Delta$. The mapping $\Delta$ can be defined as long as the colors of children edges are different from that of their parent edge. Then the image of $\Delta$ of a tree considered in the theorem just satisfies that non-root edges are colored with $[k-1]$, so that each of the first $n-2$ columns of its colored Prüfer code is an arbitrary element in $[n] \times[k-1]$. 
Theorem 8. The number of different labelled trees with vertex set $[n]$ whose edges are colored from a set of $k$ colors in such a way that any pair of edges sharing a vertex with a common parent edge have distinct colors is

$$
k(n-2) !\left(\begin{array}{c}
n k \\
n-2
\end{array}\right) .
$$

Proof. Let $T$ be a tree with the property in the statement. Following the steps for the definition of $\phi$, we can obtain an array $\sigma$ corresponding to $T$ :

$$
\sigma=\left(\begin{array}{ccccc}
a_{1} & a_{2} & \cdots & a_{n-2} & 1 \\
c_{1} & c_{2} & \cdots & c_{n-2} & c_{n-1}
\end{array}\right) .
$$

There are $k$ possible ways to choose $c_{n-1}$. Since the $c_{n-2}$ may be identical with $c_{n-1}$, the number of possible ways to choose the $(n-2)$-th column of $\sigma$ is $n k$. Since the $i$-th column of $T$ is different from the columns from the $(i+1)$-th to the $(n-2)$-th for $1 \leq i \leq n-3$, the number of possible ways to choose the $i$-th column decreases by 1 when $i$ changes from $n-2$ to 1 . So the number of such trees is

$$
k(n k)(n k-1)(n k-2) \cdots(n k-n+3)=k(n-2) !\left(\begin{array}{c}
n k \\
n-2
\end{array}\right) .
$$

\section{References}

[1] J. H. van Lint and R. M. Wilson, A Course in Combinatorics, Cambridge University Press (1992).

[2] R. P. Stanley, Enumerative Combinatorics vol. 2, Cambridge University Press (1999) 